\title{
RANDOM DETERMINANTS, MIXED VOLUMES OF ELLIPSOIDS, AND ZEROS OF GAUSSIAN RANDOM FIELDS
}

\author{
ZAKHAR KABLUCHKO AND DMITRY ZAPOROZHETS
}

\begin{abstract}
Consider a $d \times d$ matrix $M$ whose rows are independent centered non-degenerate Gaussian vectors $\xi_{1}, \ldots, \xi_{d}$ with covariance matrices $\Sigma_{1}, \ldots, \Sigma_{d}$. Denote by $\mathcal{E}_{i}$ the location-dispersion ellipsoid of $\xi_{i}: \mathcal{E}_{i}=\{\mathbf{x} \in$ $\left.\mathbb{R}^{d}: \mathbf{x}^{\top} \Sigma_{i}^{-1} \mathbf{x} \leqslant 1\right\}$. We show that

$$
\mathbb{E}|\operatorname{det} M|=\frac{d !}{(2 \pi)^{d / 2}} V_{d}\left(\mathcal{E}_{1}, \ldots, \mathcal{E}_{d}\right)
$$

where $V_{d}(\cdot, \ldots, \cdot)$ denotes the mixed volume. We also generalize this result to the case of rectangular matrices. As a direct corollary we get an analytic expression for the mixed volume of $d$ arbitrary ellipsoids in $\mathbb{R}^{d}$.

As another application, we consider a smooth centered non-degenerate Gaussian random field $X=\left(X_{1}, \ldots, X_{k}\right)^{\top}: \mathbb{R}^{d} \rightarrow \mathbb{R}^{k}$. Using Kac-Rice formula, we obtain the geometric interpretation of the intensity of zeros of $X$ in terms of the mixed volume of location-dispersion ellipsoids of the gradients of $X_{i} / \sqrt{\operatorname{Var} X_{i}}$. This relates zero sets of equations to mixed volumes in a way which is reminiscent of the well-known Bernstein theorem about the number of solutions of the typical system of algebraic equations.
\end{abstract}

\section{Main Results}

1.1. Random determinant and mixed volume of ellipsoids. Consider independent centered non-degenerate Gaussian random vectors $\xi_{1}, \ldots, \xi_{k} \in \mathbb{R}^{d}, k \leqslant d$, with covariance matrices $\Sigma_{1}, \ldots, \Sigma_{k}$. Denote by $\mathcal{E}_{i}$ the location-dispersion ellipsoid of $\xi_{i}$ :

$$
\mathcal{E}_{i}=\left\{\mathbf{x}=\left(x_{1}, \ldots, x_{d}\right)^{\top} \in \mathbb{R}^{d}: \mathbf{x}^{\top} \Sigma_{i}^{-1} \mathbf{x} \leqslant 1\right\}, \quad i=1, \ldots, k .
$$

Denote by $M$ a $k \times d$ matrix whose rows are $\xi_{1}, \ldots, \xi_{k}$.

Theorem 1.1. It holds that

$$
\mathbb{E} \sqrt{\operatorname{det}\left(M M^{\top}\right)}=\frac{(d)_{k}}{(2 \pi)^{k / 2} \kappa_{d-k}} V_{d}\left(\mathcal{E}_{1}, \ldots, \mathcal{E}_{k}, B, \ldots, B\right),
$$

where $V_{d}(\cdot, \ldots, \cdot)$ denotes the mixed volume of d convex bodies in $\mathbb{R}^{d}$ (see Sect. Q for details), $B$ is the unit ball in $\mathbb{R}^{d},(d)_{k}=d(d-1) \cdots(d-k+1)$ is the Pochhammer symbol, and $\kappa_{n}=\pi^{n / 2} / \Gamma(1+n / 2)$ denotes the volume of the unit ball in $\mathbb{R}^{n}$.

The left-hand side of (1.2) can be interpreted as the average $k$-dimensional volume of a Gaussian random parallelotope.

2010 Mathematics Subject Classification. Primary 60B20, 52A39; Secondary 60G15, 53C65.

Key words and phrases. Gaussian random determinant, Wishart matrix, Gaussian random parallelotope, mixed volumes of ellipsoids, location-dispersion ellipsoid, zeros of Gaussian random fields, Bernstein theorem, Kac-Rice formula.

The second author is partially supported by RFBR (10-01-00242), NSh-1216.2012.1, and DFG (436 RUS 113/962/0-1 R) grants. 
Corollary 1.2. In the case $k=d$ it holds that

$$
\mathbb{E}|\operatorname{det} M|=\frac{d !}{(2 \pi)^{d / 2}} V_{d}\left(\mathcal{E}_{1}, \ldots, \mathcal{E}_{d}\right) .
$$

As another direct corollary we can calculate the mixed volume of $d$ arbitrary ellipsoids in $\mathbb{R}^{d}$.

Corollary 1.3. If $\mathcal{E}_{1}, \ldots, \mathcal{E}_{d}$ are arbitrary ellipsoids defined by the symmetric positive-definite matrices $\Sigma_{1}, \ldots, \Sigma_{d}$ as in (1.1), then

$$
\begin{aligned}
V_{d}\left(\mathcal{E}_{1}, \ldots, \mathcal{E}_{d}\right) & =\frac{1}{d !} \prod_{i=1}^{d}\left(\operatorname{det} \Sigma_{i}\right)^{-1 / 2} \\
& \times \int_{\mathbb{R}^{d^{2}}}\left|\operatorname{det}\left(x_{i j}\right)\right| \prod_{i=1}^{d} \exp \left(-\frac{1}{2} \mathbf{x}_{i}^{\top} \Sigma_{i}^{-1} \mathbf{x}_{i}\right) d x_{11} \ldots d x_{d d},
\end{aligned}
$$

where

$$
\mathbf{x}_{i}=\left(x_{i 1}, \ldots, x_{i d}\right)^{\top} .
$$

The only estimate of the mixed volume of ellipsoids that we know is due to Barvinok [2]. He showed that

$$
\frac{\kappa_{d}}{3^{(d-1) / 2}} \sqrt{D_{d}\left(\Sigma_{1}, \ldots, \Sigma_{d}\right)} \leqslant V_{d}\left(\mathcal{E}_{1}, \ldots, \mathcal{E}_{d}\right) \leqslant \kappa_{d} \sqrt{D_{d}\left(\Sigma_{1}, \ldots, \Sigma_{d}\right)}
$$

where $D_{d}(\cdot, \ldots, \cdot)$ denotes the mixed discriminant of $d$ symmetric $d \times d$ matrices:

$$
D_{d}\left(A_{1}, \ldots, A_{d}\right)=\left.\frac{1}{d !} \frac{\partial^{d}}{\partial \lambda_{1} \ldots \partial \lambda_{d}} \operatorname{det}\left(\lambda_{1} A_{1}+\cdots+\lambda_{d} A_{d}\right)\right|_{\lambda_{1}=\cdots=\lambda_{d}=0} .
$$

If $\xi_{1}, \ldots, \xi_{k}$ are independent standard Gaussian vectors, then $M M^{\top}$ is a Wishart matrix, and (1.2) turns to (see [10, ,5])

$$
\mathbb{E} \sqrt{\operatorname{det}\left(M M^{\top}\right)}=\frac{(d)_{k} \kappa_{d}}{(2 \pi)^{k / 2} \kappa_{d-k}} .
$$

1.2. Intrinsic volumes. If $\xi_{1}, \xi_{2}, \ldots, \xi_{k} \in \mathbb{R}^{d}, k \leqslant d$, are identically distributed with the common covariance matrix $\Sigma$ and the location-dispersion ellipsoid $\mathcal{E}$, then (1.2) turns to

$$
\mathbb{E} \sqrt{\operatorname{det}\left(M M^{\top}\right)}=\frac{k !}{(2 \pi)^{k / 2}} V_{k}(\mathcal{E}),
$$

where $V_{k}(\cdot)$ denotes the $k$-th intrinsic volume of a convex body in $\mathbb{R}^{d}$ :

$$
V_{k}(K)=\frac{\left(\begin{array}{l}
d \\
k
\end{array}\right)}{\kappa_{d-k}} V_{d}(\underbrace{K, \ldots, K}_{k \text { times }}, B, \ldots, B) .
$$

The normalization is chosen so that $V_{k}(K)$ depends only on $K$ and not on the dimension of the surrounding space, that is, if $\operatorname{dim} K<d$, then the computation of $V_{k}(K)$ in $\mathbb{R}^{d}$ leads to the same result as the computation in the affine span of $K$. In particular, if $\operatorname{dim} K=k$, then $V_{k}(K)=\operatorname{Vol}_{k}(K)$, the $k$-dimensional volume of $K$.

It is known that $V_{1}(K)$ is proportional to the mean width of $K$ :

$$
V_{1}(K)=\frac{d \kappa_{d}}{2 \kappa_{d-1}} w(K)
$$


Taking $k=1$ in (1.3), we obtain that for any centered Gaussian vector $\xi$ with the location-dispersion ellipsoid $\mathcal{E}$ it holds that

$$
\mathbb{E}\|\xi\|=\frac{1}{\sqrt{2 \pi}} V_{1}(\mathcal{E})
$$

It was pointed out by Mikhail Lifshits that (1.4) is a special case of the following remarkable result of Sudakov.

1.3. Connection with Sudakov's result. For our purposes the following finitedimensional version of Sudakov's theorem suffices. The result in full generality can be found in [9, Proposition 14].

Proposition 1.4. For an arbitrary subset $A \subset \mathbb{R}^{d}$ we have

$$
\mathbb{E} \sup _{\mathbf{x} \in A}\langle\mathbf{x}, \eta\rangle=\frac{1}{\sqrt{2 \pi}} V_{1}(\operatorname{conv}(A)),
$$

where $\eta$ is a standard Gaussian vector in $\mathbb{R}^{d}$ and $\operatorname{conv}(A)$ is the convex hull of $A$.

Let us deduce (1.4) from (1.5). Consider a matrix $U$ such that $\Sigma=U^{-1}\left(U^{-1}\right)^{\top}$ and $U \xi$ is a standard Gaussian vector. Using (1.5) with $A=\mathcal{E}$ and $\eta=U \xi$, we get

$$
\begin{aligned}
\mathbb{E}\|\xi\|=\mathbb{E} \sup _{\|\mathbf{x}\| \leqslant 1}\langle\mathbf{x}, \xi\rangle & =\mathbb{E} \sup _{\|\mathbf{x}\| \leqslant 1}\left\langle\left(U^{-1}\right)^{\top} \mathbf{x}, U \xi\right\rangle \\
& =\mathbb{E} \sup _{\left\|U^{\top} \mathbf{x}\right\| \leqslant 1}\langle\mathbf{x}, U \xi\rangle=\mathbb{E} \sup _{\mathbf{x} \in \mathcal{E}}\langle\mathbf{x}, U \xi\rangle=\frac{1}{\sqrt{2 \pi}} V_{1}(\mathcal{E}) .
\end{aligned}
$$

Open problem: to obtain a formula generalizing (1.3) and (1.5).

1.4. Zeros of Gaussian random fields. Let $X(\mathbf{t})=\left(X_{1}(\mathbf{t}), \ldots, X_{k}(\mathbf{t})\right)^{\top}: \mathbb{R}^{d} \rightarrow$ $\mathbb{R}^{k}, k \leqslant d$, be a random field. Following Azaïs and Wschebor [1, we always assume that the following conditions hold:

(a) $X$ is Gaussian;

(b) almost surely, the function $X(\cdot)$ is of class $\mathcal{C}^{1}$;

(c) for all $\mathbf{t} \in \mathbb{R}^{d}, X(\mathbf{t})$ has a non-degenerate distribution;

(d) almost surely, if $X(\mathbf{t})=0$, then $X^{\prime}(\mathbf{t})$, the Jacobian matrix of $X(\mathbf{t})$, has the full rank.

Then, almost surely, the level set $X^{-1}(0)$ is a $\mathcal{C}^{1}$-manifold of dimension $d-k$, and for any Borel set $F$ the Lebesgue measure $\operatorname{Vol}_{d-k}\left(X^{-1}(0) \cap F\right)$ is well-defined $\left(\operatorname{Vol}_{0}(\cdot)\right.$ denotes the counting measure).

It was shown in [1, p. 177] that

$$
\mathbb{E} \operatorname{Vol}_{d-k}\left(X^{-1}(0) \cap F\right)=\int_{F} \mathbb{E}\left(\sqrt{\operatorname{det}\left(X^{\prime}(\mathbf{t}) X^{\prime}(\mathbf{t})^{\top}\right)} \mid X(\mathbf{t})=0\right) p_{X(\mathbf{t})}(0) d \mathbf{t},
$$

where $p_{X(\mathbf{t})}(\cdot)$ is a density of $X(\mathbf{t})$. Thus, the integrand in (1.6) can be interpreted as the intensity of zeros of $X$.

In this paper we consider the special case when $X$ is centered and its coordinates $X_{1}, \ldots, X_{k}$ are independent. Denote by $\mathcal{E}_{i}(\mathbf{t})$ the location-dispersion ellipsoid of $\nabla\left[X_{i}(\mathbf{t}) / \sqrt{\operatorname{Var} X_{i}(\mathbf{t})}\right]$.

Theorem 1.5. Let $X$ be a centered random field with independent coordinates defined as above and satisfying conditions $(a)-(d)$. Then

$$
\mathbb{E} \operatorname{Vol}_{d-k}\left(X^{-1}(0) \cap F\right)=\frac{(d)_{k}}{(2 \pi)^{k} \kappa_{d-k}} \int_{F} V_{d}\left(\mathcal{E}_{1}(\mathbf{t}), \ldots, \mathcal{E}_{k}(\mathbf{t}), B, \ldots, B\right) d \mathbf{t}
$$


Formula (1.7) relates zero sets of random equations to mixed volumes. In the case $k=d$ it is therefore reminiscent of the well-known fact from the algebraic geometry which we formulate in the next subsection.

1.5. Bernstein's theorem. Consider a complex polynomial in $d$ variables

$$
f\left(z_{1}, \ldots, z_{d}\right)=\sum c_{j_{1}, \ldots, j_{d}} z_{1}^{j_{1}} \ldots z_{d}^{j_{d}} .
$$

The Newton polytope of $f$ is a subset of $\mathbb{R}^{d}$ defined as

$$
\mathrm{Nw}(f)=\operatorname{conv}\left\{\left(j_{1}, \ldots, j_{d}\right) \in \mathbb{Z}^{d}: c_{j_{1}, \ldots, j_{d}} \neq 0\right\} .
$$

Let $K_{1}, \ldots, K_{d}$ be compact convex polytopes in $\mathbb{R}^{d}$ with vertexes in $\mathbb{Z}^{d}$. Consider a system of algebraic equations

$$
\left\{\begin{array}{c}
f_{1}\left(z_{1}, \ldots, z_{d}\right)=0 \\
\ldots \\
f_{d}\left(z_{1}, \ldots, z_{d}\right)=0
\end{array}\right.
$$

such that $\mathrm{Nw}\left(f_{i}\right)=K_{i}$. Bernstein showed [3] that for almost all such systems (with respect to the Lebesgue measure in the space of the coefficients of the polynomials) the number of nonzero solutions is equal to

$$
\operatorname{Vol}_{0}\left(f_{1}^{-1}(0) \cap \cdots \cap f_{d}^{-1}(0) \backslash\{\mathbf{0}\}\right)=d ! V_{d}\left(K_{1}, \ldots, K_{d}\right) .
$$

\section{Some essential tools From GeOMetry}

For the basic facts from integral and convex geometry we refer the reader to [4] and $[8$.

2.1. Mixed volumes. Consider arbitrary convex bodies $K_{1}, \ldots, K_{d} \subset \mathbb{R}^{d}$. Minkowski showed [7] that $\operatorname{Vol}_{d}\left(\lambda_{1} K_{1}+\cdots+\lambda_{d} K_{d}\right)$, where $\lambda_{1}, \ldots, \lambda_{d} \geq 0$, is a homogeneous polynomial of degree $d$ with nonnegative coefficients:

$$
\operatorname{Vol}_{d}\left(\lambda_{1} K_{1}+\cdots+\lambda_{d} K_{d}\right)=\sum_{i_{1}=1}^{d} \ldots \sum_{i_{d}=1}^{d} \lambda_{i_{1}} \ldots \lambda_{i_{d}} V_{d}\left(K_{i_{1}}, \ldots, K_{i_{d}}\right) .
$$

The coefficients $V_{d}\left(K_{i_{1}}, \ldots, K_{i_{d}}\right)$ are uniquely determined by the assumption that they are symmetric with respect to permutations of $K_{i_{1}}, \ldots, K_{i_{d}}$. The coefficient $V_{d}\left(K_{1}, \ldots, K_{d}\right)$ is called the mixed volume of $K_{1}, \ldots, K_{d}$. Differentiating (2.1), we get an alternative definition of the mixed volume:

$$
V_{d}\left(K_{1}, \ldots, K_{d}\right)=\left.\frac{1}{d !} \frac{\partial^{d}}{\partial \lambda_{1} \ldots \partial \lambda_{d}} \operatorname{Vol}_{d}\left(\lambda_{1} K_{1}+\cdots+\lambda_{d} K_{d}\right)\right|_{\lambda_{1}=\cdots=\lambda_{d}=0}
$$

For any affine transformation $L$ it holds

$$
V_{d}\left(L K_{1}, \ldots, L K_{d}\right)=|\operatorname{det} L| \cdot V_{d}\left(K_{1}, \ldots, K_{d}\right) .
$$

The following relation can also be stated:

$$
\int_{\mathbb{S}^{d-1}} V_{d-1}\left(P_{\mathbf{u}} K_{1}, \ldots, P_{\mathbf{u}} K_{d-1}\right) d \mathbf{u}=\frac{\kappa_{d-1}}{\kappa_{d}} V_{d}\left(K_{1}, \ldots, K_{d-1}, B\right),
$$

where $d \mathbf{u}$ is the surface measure on $\mathbb{S}^{d-1}$ normalized to have total mass 1 , and $P_{\mathbf{u}}$ denotes the orthogonal projection to the linear hyperplane $\mathbf{u}^{\perp}$. 
2.2. Volumes of parallelotopes. For any $A \subset \mathbb{R}^{d}$ and $\mathbf{x}_{1}, \ldots, \mathbf{x}_{k} \in \mathbb{R}^{d}$ denote by $P_{\mathbf{x}_{1}, \ldots, \mathbf{x}_{k}} A$ the orthogonal projection of $A$ to $\operatorname{span}^{\perp}\left\{\mathbf{x}_{1}, \ldots, \mathbf{x}_{k}\right\}$ (the orthogonal complement of the linear span of $\left.\mathbf{x}_{1}, \ldots, \mathbf{x}_{k}\right)$. Denote by $H_{\mathbf{x}_{1}, \ldots, \mathbf{x}_{k}}$ the parallelotope generated by the vectors $\mathbf{x}_{1}, \ldots, \mathbf{x}_{k}$. It is known that

$$
\operatorname{Vol}_{k}\left(H_{\mathbf{x}_{1}, \ldots, \mathbf{x}_{k}}\right)=\sqrt{\operatorname{det}\left(A A^{\top}\right)}
$$

where $A$ is a matrix whose rows are $\mathbf{x}_{1}, \ldots, \mathbf{x}_{k}$.

For any $\mathbf{x}_{1}, \ldots, \mathbf{x}_{d} \in \mathbb{R}^{d}$ and $k=1, \ldots, d-1$ it holds that

$$
\operatorname{Vol}_{d}\left(H_{\mathbf{x}_{1}, \ldots, \mathbf{x}_{d}}\right)=\operatorname{Vol}_{k}\left(H_{\mathbf{x}_{1}, \ldots, \mathbf{x}_{k}}\right) \operatorname{Vol}_{d-k}\left(P_{\mathbf{x}_{1}, \ldots, \mathbf{x}_{k}} H_{\mathbf{x}_{k+1}, \ldots, \mathbf{x}_{d}}\right) .
$$

2.3. Ellipsoids. There is a bijection $A \mapsto \mathcal{E}$ between $d \times d$ symmetric positivedefinite matrices and $d$-dimensional non-degenerate ellipsoids centered on the origin (see [6] for details):

$$
\mathcal{E}=\left\{\mathbf{x} \in \mathbb{R}^{d}: \mathbf{x}^{\top} A^{-1} \mathbf{x} \leqslant 1\right\}
$$

Any non-degenerate linear coordinate transformation of the form $\mathbf{x} \mapsto L \mathbf{x}$ is reflected by a change of the corresponding representing matrix $A$ to a matrix $A_{L}$ given by

$$
A_{L}=L A L^{\top} .
$$

Let $\mathcal{E}^{\prime}$ be an orthogonal projection of $\mathcal{E}$ onto an $k$-dimensional subspace with some orthonormal basis $\mathbf{x}_{1}, \ldots, \mathbf{x}_{k} \in \mathbb{R}^{d}$. Denote by $A^{\prime}$ a $k \times k$ matrix representing the ellipsoid $\mathcal{E}^{\prime}$ in this basis. If $C$ is a $k \times d$ matrix whose rows are $\mathbf{x}_{1}, \ldots, \mathbf{x}_{k}$, then

$$
A^{\prime}=C A C^{\top} \text {. }
$$

\section{Proofs}

3.1. Proof of Theorem 1.1. Case $k=d$. We proceed by induction on $d$. First let us assume that $\xi_{d}$ is a standard Gaussian vector. Denote by $\chi_{d}$ a random variable having the chi distribution with $d$ degrees of freedom and independent from $\xi_{1}, \ldots, \xi_{d-1}$. Using (2.4) and (2.5) with $k=1$ we get

$$
\begin{aligned}
\mathbb{E}|\operatorname{det} M|=\mathbb{E} \operatorname{Vol}_{d}\left(H_{\xi_{1}, \ldots, \xi_{d}}\right) & =\int_{\mathbb{S}^{d-1}} \mathbb{E} \operatorname{Vol}_{d}\left(H_{\xi_{1}, \ldots, \xi_{d-1}, \chi_{d} \mathbf{u}}\right) d \mathbf{u} \\
& =\mathbb{E} \chi_{d} \int_{\mathbb{S}^{d-1}} \mathbb{E} \operatorname{Vol}_{d-1}\left(P_{\mathbf{u}} H_{\xi_{1}, \ldots, \xi_{d-1}}\right) d \mathbf{u} \\
& =\frac{d \kappa_{d}}{\sqrt{2 \pi} \kappa_{d-1}} \int_{\mathbb{S}^{d-1}} \mathbb{E} \operatorname{Vol}_{d-1}\left(H_{P_{\mathbf{u}} \xi_{1}, \ldots, P_{\mathbf{u}} \xi_{d-1}}\right) d \mathbf{u} .
\end{aligned}
$$

It follows from (2.7) that $P_{\mathbf{u}} \xi_{i}$ has a location-dispersion ellipsoid $P_{\mathbf{u}} \mathcal{E}_{i}$. By the induction assumption,

$$
\mathbb{E} \operatorname{Vol}_{d-1}\left(H_{P_{\mathbf{u}} \xi_{1}, \ldots, P_{\mathbf{u}} \xi_{d-1}}\right)=\frac{(d-1) !}{(2 \pi)^{(d-1) / 2}} V_{d-1}\left(P_{\mathbf{u}} \mathcal{E}_{1}, \ldots, P_{\mathbf{u}} \mathcal{E}_{d-1}\right)
$$

Combining the latter two relations with (2.3), we obtain

$$
\mathbb{E}|\operatorname{det} M|=\frac{d !}{(2 \pi)^{d / 2}} V_{d}\left(\mathcal{E}_{1}, \ldots, \mathcal{E}_{d-1}, B\right) .
$$

If $\xi_{d}$ is an arbitrary non-degenerate Gaussian vector, then there exists a linear transformation $L$ such that $L \xi_{d}$ is a standard Gaussian vector. It follows from (2.6) 
that $L \mathcal{E}_{i}$ is the location-dispersion ellipsoid of $L \xi_{i}$, and in particular $L \mathcal{E}_{d}=B$. Applying (3.1) to the matrix $L M^{\top}$ and using (2.2), we get

$$
\begin{aligned}
\mathbb{E}|\operatorname{det} M|=|\operatorname{det} L|^{-1} \mathbb{E}\left|\operatorname{det} L M^{\top}\right| & =\frac{d !}{(2 \pi)^{d / 2}}|\operatorname{det} L|^{-1} V_{d}\left(L \mathcal{E}_{1}, \ldots, L \mathcal{E}_{d-1}, B\right) \\
& =\frac{d !}{(2 \pi)^{d / 2}} V_{d}\left(\mathcal{E}_{1}, \ldots, \mathcal{E}_{d-1}, \mathcal{E}_{d}\right) .
\end{aligned}
$$

3.2. Proof of Theorem 1.1. Case $k<d$. Consider a $d \times d$ matrix $M^{\prime}$ whose first $k$ rows form the matrix $M$ and the last $d-k$ rows are independent standard Gaussian vectors $\xi_{k+1}, \ldots, \xi_{d}$ (independent from $M$ ). By the previous case,

$$
\mathbb{E}\left|\operatorname{det} M^{\prime}\right|=\frac{d !}{(2 \pi)^{d / 2}} V_{d}\left(\mathcal{E}_{1}, \ldots, \mathcal{E}_{k}, B, \ldots, B\right) .
$$

On the other hand, by (2.5),

$$
\begin{aligned}
\mathbb{E}\left|\operatorname{det} M^{\prime}\right|=\mathbb{E} \operatorname{Vol}_{d}\left(H_{\xi_{1}, \ldots, \xi_{d}}\right) & =\mathbb{E} \operatorname{Vol}_{k}\left(H_{\xi_{1}, \ldots, \xi_{k}}\right) \operatorname{Vol}_{d-k}\left(P_{\xi_{1}, \ldots, \xi_{k}} H_{\xi_{k+1}, \ldots, \xi_{d}}\right) \\
& =\mathbb{E} \sqrt{\operatorname{det}\left(M M^{\top}\right)} \mathbb{E} \operatorname{Vol}_{d-k}\left(H_{\eta_{1}, \ldots, \eta_{d-k}}\right),
\end{aligned}
$$

where $\eta_{1}, \ldots, \eta_{d-k}$ are independent standard Gaussian vectors in $\mathbb{R}^{d-k}$. By the previous case,

$$
\mathbb{E} \operatorname{Vol}_{d-k}\left(H_{\eta_{1}, \ldots, \eta_{d-k}}\right)=\frac{(d-k) !}{(2 \pi)^{(d-k) / 2}} \kappa_{d-k} .
$$

Combining the latter three relations completes the proof.

3.3. Proof of Theorem 1.5. First we suppose that $X_{j}$ has a unit variance: $\operatorname{Var} X_{j}(\mathbf{t}) \equiv 1$ for all $j=1, \ldots, k$. Differentiating the relation $\mathbb{E} X_{j}(\mathbf{t}) X_{j}(\mathbf{t})=1$ with respect to $t_{i}$, we obtain

$$
\mathbb{E} \frac{\partial X_{j}}{\partial t_{i}}(\mathbf{t}) X_{j}(\mathbf{t})=0,
$$

which, together with the independence of the coordinates of $X$, implies that $X^{\prime}(\mathbf{t})$ and $X(\mathbf{t})$ are independent. This means that conditioning on $X(\mathbf{t})=0$ in (1.6) may be dropped. To complete the proof of the theorem in the case $\operatorname{Var} X_{j}(\mathbf{t}) \equiv 1$ it remains to combine (1.6) with (1.2).

To cover the general case, it suffices to note that $X_{j} / \sqrt{\operatorname{Var} X_{j}}$ has the same zero set as $X_{j}$.

Acknowledgments. We are grateful to Mikhail Lifshits for brining our attention to Sudakov's result.

\section{REFERENCES}

[1] J.M. Azaïs and M. Wschebor, Level sets and extrema of random processes and fields, Wiley, 2009.

[2] A. Barvinok, Computing mixed discriminants, mixed volumes, and permanents, Discrete Comput. Geom. 18 (1997), no. 2, 205-237.

[3] D.N. Bernshtein, The number of roots of a system of equations, Funct. Anal. Appl. 9 (1975), no. 3, 183-185.

[4] Yu. D. Burago and V. A. Zalgaller, Geometric inequalities, Grundlehren der Mathematischen Wissenschaften, vol. 285, Springer-Verlag, Berlin, 1988, Translated from the Russian.

[5] N.R. Goodman, The distribution of the determinant of a complex Wishart distributed matrix, Ann. Math. Statist. 34 (1963), no. 1, 178-180. 
[6] W.C. Karl, G.C. Verghese, and A.S. Willsky, Reconstructing ellipsoids from projections, CVGIP: Graphical Model and Image Processing 56 (1994), no. 2, 124-139.

[7] H. Minkowski, Theorie der konvexen Körper, insbesondere Begründung ihres Oberflächenbegriffs, In: Gesammelte Abhandlungen, Vol. 2, p. 131-229.

[8] R. Schneider and W. Weil, Stochastic and integral geometry, Springer-Verlag, 2008.

[9] V. N. Sudakov, Geometric problems in the theory of infinite-dimensional probability distributions, Proc. Steklov Inst. Math. 1979, no. 2, 1-178, Translation of Tr. Mat. Inst. Steklova 141 (1976).

[10] S.S. Wilks, Moment-generating operators for determinants of product moments in samples from a normal system, Ann. of Math. 35 (1934), no. 2, 312-340.

Zakhar Kabluchko, Institute of Stochastics, Ulm University, Helmholtzstr. 18, 89069 Ulm, Germany

E-mail address: zakhar.kabluchko@uni-ulm.de

Dmitry Zaporozhets, St. Petersburg Department of Steklov Institute of Mathematics, Fontanka 27, 191011 St. Petersburg, Russia

E-mail address: zap1979@gmail.com 